\title{
Rational suicide and dementia
}

\author{
B Draper \\ Conjoint Professor, School of Psychiatry, University of New South Wales, Sydney, Australia
}

TITLE Alzheimer disease and pre-emptive suicide

AUTHOR Davis DS

JOURNAL J Med Ethics 2013. Epub 2013 Jul 10. http://dx.doi.org//0.1I36/ medethics-2012-101022

DECLARATION OF INTERESTS No conflicts of interest declared.

\author{
Correspondence to B Draper \\ Academic Department for \\ Old Age Psychiatry \\ Euroa Centre, Prince of \\ Wales Hospital \\ Randwick, NSW 203 I, Australia \\ tel +(6I) 293823753 \\ e-mail b.draper@unsw.edu.au
}

\section{SUMMARY}

In this opinion piece, Davis proposes that suicide is a reasonable response to impending dementia and that the move towards pre-symptomatic diagnosis of Alzheimer's disease (AD) now makes rational suicide a more feasible option for those so inclined. Three broad reasons are proposed for earlier death through rational suicide: to conserve financial assets; to reduce family burden of care; and to allow people to end their lives 'with a final chapter that is consonant with the narrative as a whole'. There are numerous barriers to ending one's life to avoid living with dementia. Even though Davis admits that few types of dementia have accurate genetic prediction, clinicians are criticised for their paternalistic attitudes in not doing genetic tests and divulging results to people who indicate an interest in rational suicide.

The 'Catch-22' dilemma with dementia is discussed people don't want to die while they remain competent, but they are unable to suicide when they have lost capacity. In jurisdictions where assisted suicide is legal, the person must be competent to make the decision. Advance directives can be ignored and many authorities believe that'they lack the full moral force of contemporary choices and that respect for precedent autonomy is misdirected or downright unethical'. Davis does not accept these viewpoints but concedes that they are difficult to refute and add further weight to the notion of pre-emptive suicide for those so inclined.

The increasing accuracy and availability of biomarkers may herald an era when the onset of dementia can be predicted in time for the person to make a rational decision to suicide. Davis believes that 'direct to consumer' genetic testing bypasses the clinician barrier. She states that 'it has become increasingly possible to diagnose $A D$ by laboratory tests' and mentions the revised AD research criteria that incorporate biomarkers for pre-symptomatic diagnosis. Finally, Davis argues that gatekeepers such as researchers, clinicians and policymakers need to acknowledge the option of pre-emptive suicide when deciding how to allocate resources and provide information about biomarkers to research participants and patients so that they can make an exit strategy if desired.

\section{COMMENTARY}

There is divided opinion about the rights and wrongs of rational suicide,' but that debate is not the focus of this article. The two key issues raised are the accuracy of biomarkers in pre-symptomatic $A D$ or other types of dementia and about the obligations of clinicians and researchers to provide this biomarker information to patients and research participants.

Having lucidly discussed the dilemma that people who desire rational suicide often want to delay their decision so as not to miss out on 'precious good years', Davis ignores the fundamental issue that biomarkers do not address this conundrum. Some biomarkers address dementia risk, and others imminence, but these are not measures of quality of life or decision-making capacity. ${ }^{2}$ Having a biomarker result that basically informs the person that at some point in the next few years, symptoms of $A D$ might appear, or get worse, is not going to help most individuals to make a decision about when to die by suicide if they want to live those 'precious good years'. However, it might increase the risk of suicide in those whose decision-making capacity is temporarily affected by the news - and while that might be understandable, it is not rational suicide.' Guidelines for the provision of genetic and biomarker information to people at risk of dementia involve appropriate counselling but this is not going to happen with direct to consumer genetic testing.'

Clinicians have ethical obligations to respect human life. The Declaration of Helsinki charges medical researchers with the responsibility to protect research participants including not passing on potentially misleading research information that has yet to be validated. ${ }^{3}$ There are also broader ethical responsibilities that clinicians and researchers have to consider as the impact of suicide upon families and health professionals can be profound. ${ }^{4}$ Indeed, it has recently been argued that from a moral viewpoint, suicide cannot be evaluated solely in terms of personal autonomy; communal values also play a role. ${ }^{5}$ It is little wonder that clinicians and researchers might feel uneasy about passing on information that may be inaccurate and that result in the person's suicide. 


\title{
REFERENCES
}

I Draper B, Peisah C, Snowdon J et al. Early dementia diagnosis and the risk of suicide and euthanasia. Alzheimers Dement 2010; 6:7582. http://dx.doi.org//0.1016/j.jalz.2009.04.1229

2 Galasko D, Golde TE. Biomarkers for Alzheimer's disease in plasma, serum and blood - conceptual and practical problems. Alzheimer's Research \& Therapy 2013; 5: 10. http://dx.doi.org/I0.1 I86/alzrtl64

3 World Medical Association. WMA Declaration of Helsinki - ethical principles for medical research involving human subjects [Internet]. France: WMA. Available from: http://www.wma.net/ en/30publications/I0policies/b3/
4

Sveen CA, Walby FA. Suicide survivors' mental health and grief reactions: a systematic review of controlled studies. Suicide Life Threat Behav 2008; 38:13-29. http://dx.doi.org/I0.152I/suli.2008.38.I.I3

5 Pies R. Is suicide immoral? Psychiatric Times 20I4; 3I:I-4.

\section{Clinical opinion}

J R Coll Physicians Edinb 2014; 44:131-2 http://dx.doi.org/ I0.4997/JRCPE.2014.209 (C) 2014 Royal College of Physicians of Edinburgh

\section{Associations and clinical significance in meta- analysis: when are the deductions too presumptive?}

\author{
RJ MacFadyen \\ Director of Internal Medicine/Staff Cardiologist, Ballarat Health and University of Melbourne, Ballarat, Australia
}

TITLE Off hour presentation and outcomes in patients with acute myocardial infarction: systematic review and meta-analysis

AUTHORS Sorita A, Ahmed A, Starr S et al.

JOURNAL BMJ 20I4; 348:f7393. http://dx.doi.org/l0.। I36/bmj.f7393

DECLARATION OF INTERESTS No conflicts of interest declared.

\author{
Correspondence to RJ MacFadyen, \\ Ballarat Health and University of \\ Melbourne, Ballarat, VIC 3350 \\ Australia
}

e-mail robertma@bhs.org.au

\section{SUMMARY}

This meta-analysis of publications over an approximate ten-year period from 2002-12 looked at mortality after coronary presentations. A total of 487 reports were studied and 48 were assessed for the impact of nominal 'off hours' (night and weekends) presentation for catheter-based revascularisation for ST elevation myocardial infarction (STEMI). Smaller studies tended to show increased mortality off hours, larger samples little or no adverse effect. The net impact, using a random effect meta-analysis model, was a statistically significant $2-4 \%$ mortality excess. This was linked by the authors to an increase in hospital 'door to balloon' times.

Data on time to recognition and hospital presentation were not available but were said to be shorter off hours as seen in other publications. More adverse effects were seen off hours in non-North American centres and was associated with a reduced door to balloon time in North American registries.

The authors go on to suggest these observations have implications for the assessment of quality of care by hospitals (and staff) and should guide 'value based purchasing' on the basis of off hours performance. The limitations - lack of randomisation, inconsistent definition of 'off hours', high heterogeneity, publication bias, overlapping patients - were all felt to be offset by the statistical power of analysis (more than one million patients from the 48 publications included). The authors considered that previous failed attempts to confirm the adverse off hours effect on mortality were due to a lack of statistical power.

\section{COMMENTARY}

Most practitioners have accepted that adverse outcomes can follow out-of-hours or weekend management in communities or hospitals. This rightly attracts much political attention, yet where we do not understand the basic mechanisms; the causes of any adverse clincial effects are unlikely to be generalisable. Does this further analysis in STEMI add anything new?

The presumption that every intervention can be, or should be, performed in the same way, day or night, regardless of individual circumstances is a basic error.That biological variance can be explained via statistical association in a meta-analysis is also erroneous. Where numbers are this large, statistical significance can be guaranteed. Plausible explanations can be suggested (staff/ facilities don't respond quickly or are not available), but they remain speculative. 